\section{Mini Review}

\section{*Corresponding author}

Erika Rabelo Forte de Siqueira, MD, PhD

Department of Gastroenterology

University of Pernambuco School of

Medicine

Pernambuco, Brazil

Liver Institute of Pernambuco

Pernambuco, Brazil

Tel. (+5581) 30352050

E-mail: erikarfs@gmail.com

\section{Volume 1 : Issue 2}

Article Ref. \#: 1000LROJ1107

\section{Article History}

Received: July $10^{\text {th }}, 2015$

Accepted: July $27^{\text {th }}, 2015$

Published: July $29^{\text {th }}, 2015$

\section{Citation}

de Siqueira ERF, Pereira LMMB, Sanyal AJ. Cardiovascular disease and NASH. Liver Res Open J. 2015 1(2): 41-44. doi: 10.17140/LROJ-1107

\title{
Cardiovascular Disease and NASH
}

\section{Erika Rabelo Forte de Siqueira ${ }^{1,2^{*}}$, Leila Maria Moreira Beltrao Pereira ${ }^{1,2}$ and Arun Jayant Sanyal ${ }^{3}$}

${ }^{1}$ Department of Gastroenterology, University of Pernambuco School of Medicine, Pernambuco, Brazil

${ }^{2}$ Liver Institute of Pernambuco, Pernambuco, Brazil

${ }^{3}$ Division of Gastroenterology, Hepatology and Nutrition, Department of Internal Medicine, Virginia Commonwealth University School of Medicine, Richmond, VA, USA

\section{ABSTRACT}

Non-alcoholic fatty liver disease (NAFLD) and Non-alcoholic steatohepatitis (NASH) are associated with cardiovascular events and Metabolic Syndrome (MetS). NAFLD is considered to be a hepatic manifestation of MetS and has become an important public health issue because of its high prevalence. It is currently being considered an independent Cardiovascular disease (CVD) risk factor. In this clinical review, we will briefly review the mechanisms linking NAFLD to the complement system, endothelial dysfunction and the atherosclerosis.

KEYWORDS AND ABBREVIATIONS: NAFLD: Non-alcoholic fatty liver disease; NASH: Nonalcoholic steatohepatitis; CVD: Cardiovascular disease; MetS: Metabolic Syndrome: AF: Atrial Fibrillation; CAC: Coronary Artery Calcium; CT: Computed Tomography; FFAs: Free Fatty Acids; LDL-C: Low-density Lipoprotein-Cholesterol; HMGCR: HMG CoA Reductase; VLDL: Very-low-density lipoprotein; CAD: Coronary Artery Disease.

\section{INTRODUCTION}

Nonalcoholic fatty liver disease (NAFLD) has become a predominant cause of chronic liver disease in many countries. Excess body weight predisposes individuals to chronic diseases such as cardiovascular disease (CVD), Type 2 Diabetes Mellitus (T2DM) and NAFLD. NAFLD and cardiovascular disease is obesity related MetS and it is increasing virtually in all age groups worldwide. ${ }^{1}$ Approximately $10-30 \%$ have the potentially progressive form of NAFLD to Non-alcoholic steatohepatitis (NASH), which is associated with hepatocellular injury, inflammation and ultimately resulting in cirrhosis in $20-30 \% .^{2-4}$

A population-based study of 980 NAFLD patients and 6,594 controls followed long term (mean: 8.7 years) showed that NAFLD patients had significantly increased all-cause mortality and cardiovascular mortality, especially in the 45-54 years age group. ${ }^{5}$ Another study that linked NHANES III participants to follow-up mortality data showed that cardiovascular disease was the leading cause of death in patients with NAFLD. ${ }^{6}$

Several evidences support the association of NAFLD/NASH and the occurrence of cardiovascular events, such as increased carotid intima-media thickness, increased coronary artery calcification, impaired flow-mediated vasodilatation and arterial stiffness independent of traditional risk factors and MetS. ${ }^{7-9}$

The finding that NAFLD is associated with an increased risk of Atrial Fibrillation (AF) in people without evidence of co-existing valvular heart disease supports the assertion that NAFLD may also be an emerging risk factor for cardiac arrhythmias. ${ }^{10,11}$ Another marker of early coronary atherosclerosis is association of NAFLD and increased Coronary Artery Calcium (CAC) score on cardiac Computed Tomography (CT). ${ }^{12}$ 
In this clinical review, we will briefly review the mechanisms linking NAFLD to the risk of cardiovascular events.

\section{MECHANISMS LINKING NAFLD WITH CARDIOVASCULAR EVENTS}

Data in the literature are still controversial regarding the increased cardiovascular risk with NASH. A large North American database reported a higher prevalence of CVD risk factors and events in patient with NAFLD in comparison with those without. Nonetheless, the rate of CVD mortality was not high in subjects with NAFLD. ${ }^{13}$ Previous studies have already demonstrated the presence of NAFLD independently increased the risk for Coronary Artery Disease (CAD) and NAFLD was more commonly found in patients as the extent of CAD increased $(\mathrm{P}=0.001) .{ }^{14}$ It has also been shown that risk, after adjustment for age, sex, race, ethnicity, body mass index, and hyperinsulinemia, children with MetS had 5.0 times the odds of having NAFLD as overweight and obese children without MetS. ${ }^{15}$

In another prospective, Japanese study of 1221 apparently healthy subjects, patients with NAFLD showed an increased incidence of CVD events $(1.0 \%$ vs. $5.2 \% ; \mathrm{P}<0.001)$ and NAFLD emerged as an independent predictor of CVD. ${ }^{16}$ However, there were no differences in the incidence of fatal CVD, non-fatal myocardial infarction, and coronary revascularization after the follow up. ${ }^{17}$

The CVD is characterized by critically narrowing (stenosis) or occlusion (atherothrombosis) of blood vessels. Key processes in CVD are endothelial dysfunction, atherosclerosis, and impaired regulation of coagulation and fibrinolysis. The complement system may be involved in all these processes based on its immune, inflammatory and metabolic functions. ${ }^{18}$

Furthermore, systemic complement levels may be involved in coagulation and fibrinolysis, which together with endothelial dysfunction and atherosclerosis result in cardiovascular disease. ${ }^{18}$ The complement-C3 has shown independent associations with insulin resistance, liver dysfunction and in the risk of MetS and T2DM. ${ }^{19,20}$

Circulating levels of several inflammatory markers (Creactive protein, interleukin-6, monocyte chemotactic protein 1 , and TNF-a), procoagulant factors (plasminogen activator inhibitor 1, fibrinogen, and factor VII), and oxidative stress markers are highest in patients with NASH independent of obesity and other potentially confounding factors. ${ }^{21}$ NAFLD seems to be not simply a marker of cardiac and arrhythmogenic complications but also may play a part in their pathogenesis possibly via atherogenic dyslipidemia and the hepatic secretion of several pathogenic mediators. ${ }^{22,23}$

Central obesity can provoke inflammation and insulin resistance in adipose tissue and the release of proinflammatory adipokines and Free Fatty Acids (FFAs). Relation of hepatic steatosis to atherogenic dyslipidemia results from previous studies that liver fat is associated with an increased number of higher Low-density Lipoprotein-Cholesterol (LDL-C), in special the small dense LDL (sdLDL) particles, which have higher atherogenic properties than larger less dense LDL-C particles. ${ }^{24-26}$

These lipoproteins are associated with increased activity of hepatic lipase favoring the production of sdLDL particles. ${ }^{27}$ Patients with NASH can have the transcriptional regulation of proatherogenic genes altered and it is associated with the activation of molecular events that may also be responsible for the local production of mediators or modifiers of circulatory homeostasis. $^{28}$

In particular, NASH presents a distinct panel of regulatory genes which are dysregulated compared to the control and subjects with simple steatosis. Increased activation of genes involved in cholesterol biosynthesis and metabolism by Sterol Regulatory Element-binding Protein-2 (SREBP-2), the principal transcriptional activator of the HMG CoA Reductase (HMGCR), the metabolic pathway that produces cholesterol and other isoprenoids, is a key factor driving both the LDL-C and accumulation of hepatic free cholesterol..$^{29,30}$

NAFLD is associated with increased Very-low-density lipoprotein (VLDL) particle concentration and apolipoprotein $\mathrm{b}$ (ApoB), sdLDL particle concentration and cholesterol content. The triglyceride levels, VLDL particle concentration and size as well as ApoB were directly related to the degree of fasting insulin levels which is consistent with the known effects of insulin on triglyceride synthesis. ${ }^{31}$

The atherogenecity of the increase in sdLDL is likely to be further compounded by our previously noted decrease in LDL receptor expression in NASH. ${ }^{29}$ From a cardiovascular point of view the direct relation between LDL-C and HMGCR expression suggests that the liver disease contributes to LDL-mediated cardiovascular risk. ${ }^{29}$

\section{CONCLUSION}

In this context, the liver represents also a contributor to systemic inflammatory changes, insulin resistance and hyperlipidemia determining a progression of vascular diseases and atherosclerosis. NAFLD/NASH are associated with an increased risk of incident cardiovascular disease, independently of the traditional risk factors. Therefore, a multidisciplinary approach to patients with multiple risk factors including MetS and T2DM is required to monitor for cardiovascular and liver complications.

\section{DECLARATION OF INTEREST}

The authors declare that they have no competing interests. 


\section{LIVER RESEARCH}

\section{REFERENCES}

1. Masuoka HC, Chalasani N. Nonalcoholic fatty liver disease: an emerging threat to obese and diabetic individuals. Ann $N Y$ Acad Sci. 2013; 1281: 106-122. doi: 10.1111/nyas. 12016

2. Wree A, Broderick L, Canbay A, et al. From NAFLD to NASH to cirrhosis-new insights into disease mechanisms. Nat Rev Gastroenterol Hepatol. 2013; 10: 627-636. doi: 10.1038/ nrgastro.2013.149

3. Williams CD, Stengel J, Asike MI, et al. Prevalence of nonalcoholic fatty liver disease and nonalcoholic steatohepatitis among a largely middle-aged population utilizing ultrasound and liver biopsy: a prospective study. Gastroenterology. 2011; 140: 124-131. doi: 10.1053/j.gastro.2010.09.038

4. Wong VW, Wong GL, Choi PC, et al. Disease progression of non-alcoholic fatty liver disease: a prospective study with paired liver biopsies at 3 years. Gut. 2010; 59: 969-974. doi: 10.1136/ gut.2009.205088

5. Dunn W, Xu R, Wingard DL, et al. Suspected nonalcoholic fatty liver disease and mortality risk in a population-based cohort study. Am J Gastroenterol. 2008; 103: 2263-2271. doi: 10.1111/j.1572-0241.2008.02034.x

6. Ong JP, Pitts A, Younossi ZM. Increased overall mortality and liver-related mortality in non-alcoholic fatty liver disease. J Hepatol. 2008; 49: 608-612. doi: 10.1016/j.jhep.2008.06.018

7. Kuang-Chun Hu, Horng-Yuan Wang, Sung-Chen Liu, et al. Nonalcoholic fatty liver disease: updates in noninvasive diagnosis and correlation with cardiovascular disease. World J Gastroenterol. 2014; 20(24): 7718-7729. doi: 10.3748/wjg.v20. i24.7718

8. Anstee QM, Targher G, Day CP. Progression of NAFLD to diabetes mellitus, cardiovascular disease or cirrhosis. Nat Rev Gastroenterol Hepatol. 2013; 10: 330-344. doi: 10.1038/nrgastro.2013.41

9. Oni ET, Agatston AS, Blaha MJ, et al. A systematic review: burden and severity of subclinical cardiovascular disease among those with nonalcoholic fatty liver; should we care? Atherosclerosis. 2013; 230: 258-267. doi: 10.1016/j.atherosclerosis.2013.07.052

10. Targher G, Mantovani A, Pichiri I, et al. Non-alcoholic fatty liver disease is associated with an increased prevalence of atrial fibrillation in hospitalized patients with type 2 diabetes. Clin $\mathrm{Sci}$ (Lond). 2013; 125(6): 301-309. doi: 10.1042/CS20130036

11. Targher G, Valbusa F, Bonapace S, et al. Non-alcoholic fatty liver disease is associated with an increased incidence of atrial fibrillation in patients with type 2 diabetes. PLoS One. 2013; 8: 57183. doi: 10.1371/journal.pone.0057183

12. Abdulla J, Asferg C, Kofoed KF. Prognostic value of absence or presence of coronary artery disease determined by 64-slice computed tomography coronary angiography a systematic review and meta-analysis. Int $J$ Cardiovasc Imaging. 2011; 27 : 413-420. doi: 10.1007/s10554-010-9652-x

13. Stepanova M, Younossi ZM. Independent association between nonalcoholic fatty liver disease and cardiovascular disease in the US population. Clin Gastroenterol Hepatol. 2012; 10: 646-650. doi: 10.1016/j.cgh.2011.12.039

14. Arslan U, Türkoğlu S, Balcioğlu S, et al. Association between nonalcoholic fatty liver disease and coronary artery disease. Coron Artery Dis. 2007; 18(6): 433-436.

15. Schwimmer JB, Pardee PE, Lavine JE, et al. Cardiovascular risk factors and the metabolic syndrome in pediatric nonalcoholic fatty liver disease. Circulation. 2008; 118(3): 277-283. doi: 10.1161/CIRCULATIONAHA.107.739920

16. Hamaguchi M, Kojima T, Takeda N, et al. Nonalcoholic fatty liver disease is a novel predictor of cardiovascular disease. World J Gastroenterol. 2007; 13: 1579-1584. doi: 10.3748/wjg. v13.i10.1579

17. Wong VW, Wong GL, Yip GW, et al. Coronary artery disease and cardiovascular outcomes in patients with nonalcoholic fatty liver disease. Gut. 2011; 60: 1721-1727. doi: 10.1136/ gut.2011.242016

18. Hertle E, Stehouwer CDA, van Greevenbroek MMJ. The complement system in human cardiometabolic disease. Molecular Immunology. 2014; 61: 135-148. doi: 10.1016/j.molimm.2014.06.031

19. Bellentani S, Scaglioni F, Marino M, et al. Epidemiology of non-alcoholic fatty liver disease. Dig Dis. 2010; 28: 155-161. doi: $10.1159 / 000282080$

20. Speidl WS, Kastl SP, Huber K, et al. Complement in atherosclerosis: friend or foe? J Thromb Haemost. 2011; 9: 428-440. doi: $10.1111 /$ j. 1538-7836.2010.04172.x

21. Targher G, Day CP, Bonora E. Risk of cardiovascular disease in patients with nonalcoholic fatty liver disease. $N$ Engl J Med. 2011; 363(14): 1341-1350. doi: 10.1056/NEJMra0912063

22. Sinner MF, Wang N, Fox CS, et al. Relation of circulating liver transaminase concentrations to risk of new-onset atrial fibrillation. Am J Cardiol. 2013; 111: 219-224. doi: 10.1016/j.am- 


\section{LIVER RESEARCH}

jcard.2012.09.021

23. Stefano Ballestri, Amedeo Lonardo, Stefano Bonapace, et al. Risk of cardiovascular, cardiac and arrhythmic complications in patients with non-alcoholic fatty liver disease. World J Gastroenterol. 2014; 20(7): 1724. doi: 10.3748/wjg.v20.i7.1724

24. Blake GJ, Otvos JD, Rifai N, et al. Low-density lipoprotein particle concentration and size as determined by nuclear magnetic resonance spectroscopy as predictors of cardiovascular disease in women. Circulation. 2002; 106: 1930-1937. doi: 10.1161/01.CIR.0000033222.75187.B9

25. Cromwell WC, Otvos JD. Low-density lipoprotein particle number and risk for cardiovascular disease. Curr Atheroscler Rep. 2004; 6: 381-387.

26. Toledo FGS, Sniderman AD, Kelley DE. Influence of hepatic steatosis (fatty liver) on severity and composition of dyslipidemia in type 2 diabetes. Diabetes Care. 2006; 29: 1845-1850. doi: $10.2337 / \mathrm{dc} 06-0455$

27. Rashid S, Watanabe T, Sakaue T, et al. Mechanisms of HDLlowering in insulin resistant, hypertriglyceridemic states: the combined effect of HDL triglyceride enrichment and elevated hepatic lipase activity. Clin Biochem. 2003; 36: 421-429. doi: 10.1016/S0009-9120(03)00078-X

28. Sookoian S, Gianotti TF, Rosselli MS, et al. Liver transcriptional profile of atherosclerosis- related genes in human nonalcoholic fatty liver disease. Atherosclerosis. 2011; 218(2): 378385. doi: 10.1016/j.atherosclerosis.2011.05.014

29. Hae-Ki Min, Ashwani Kapoor, Michael Fuchs, et al. Increased hepatic synthesis and dysregulation of cholesterol metabolism is associated with the severity of nonalcoholic fatty liver disease. Cell Metabolism. 2012; 15: 665-674. doi: 10.1016/j. cmet.2012.04.004

30. Horton JD, Shimomura I, Brown MS, Hammer RE, Goldstein JL, Shimano H. Activation of cholesterol synthesis in preference to fatty acid synthesis in liver and adipose tissue of transgenic mice overproducing sterol regulatory element-binding protein-2. J Clin Invest. 1998; 101: 2331-2339. doi: 10.1172/ JCI2961

31. Siddiqui MS, Fuchs M, Idowu M, et al. The histologic severity and progressionto cirrhosis impacts atherogenic lipoprotein profile in nonalcoholic fatty liver disease. Clin Gastroenterol Hepatol. 2014. 